Voix et Images

volxetimages

\title{
Langue et littérature en territoires subjugués
}

\section{Robert Dion}

Volume 32, numéro 2 (95), hiver 2007

Féminin/Masculin. Jeux et transformations

URI : https://id.erudit.org/iderudit/016320ar

DOI : https://doi.org/10.7202/016320ar

Aller au sommaire du numéro

\section{Éditeur(s)}

Université du Québec à Montréal

\section{ISSN}

0318-9201 (imprimé)

1705-933X (numérique)

Découvrir la revue

Citer ce compte rendu

Dion, R. (2007). Compte rendu de [Langue et littérature en territoires

subjugués]. Voix et Images, 32(2), 155-159. https://doi.org/10.7202/016320ar d'utilisation que vous pouvez consulter en ligne.

https://apropos.erudit.org/fr/usagers/politique-dutilisation/ 
R E C H E R C H E

Langue et littérature en territoires subjugués
+++

ROBERT DION

Université du Québec à Montréal

Il est des questions de recherche qui ressurgissent périodiquement, qui semblent nous hanter autant que nous les hantons, et qui néanmoins nous surprennent toujours : nous croyions tout savoir, avoir tout lu, et nous découvrons qu'il restait en fait beaucoup à dire et à penser.

C'est l'impression que j'ai eue à la lecture de La langue de papier. Spéculations linguistiques au Québec (1957-1977) de Karim Larose ${ }^{1}$. S'il est un champ de recherche où l'on pourrait croire qu'il n'y a plus guère à glaner, c'est bien celui-là. Pourtant, l'auteur nous arrive avec un fort volume, version non pas allégée d'une thèse de doctorat, comme de coutume, mais au contraire augmentée d'une centaine de pages. Je ne dirai pas que l'ouvrage n'est pas parfois bavard; et même si Larose prend bien soin, en introduction, de baliser son terrain de recherche et de reconnaître sa dette envers ses devanciers, on a parfois l'impression qu'il repasse où d'autres l'ont précédé et qu'il aurait pu, à certains moments, aller plus directement au but, à son but, qui est « de documenter, de décrire et d'analyser le changement de paradigme linguistique advenu à la fin des années 1950 au Québec» (14). Mais je ne me suis pas du tout ennuyé en sa compagnie, malgré la linéarité du parcours chronologique et le caractère çà et là très «à ras de texte» du commentaire.

Après quelques pages d'ouverture désarçonnantes, éloignées de l'objet et écrites dans un style qui ne se retrouvera plus par la suite, Larose nous conduit au cœur de sa méthode, qui consiste à tirer le fil, au sein du corpus foisonnant des interventions des intellectuels et des écrivains au cours des années qui vont du Congrès de la refrancisation (1957) à l'adoption de la Charte de la langue française (1977), d'une appréhension expressiviste de la langue, qu'il saisit dans son opposition à des visions utilitaristes et instrumentalistes. Identifié par Charles Taylor, le paradigme expressiviste est celui qui détermine «la conception du Sujet la plus répandue à l'époque moderne, où l'expression de chaque être humain est considérée comme un processus créateur qui introduit dans le monde quelque chose d'encore

$$
++
$$

1 Karim Larose, La langue de papier. Spéculations linguistiques au Québec (1957-1977), Montréal, Les Presses de l'Université de Montréal, coll. «Espace littéraire», 2004, 451 p. 
inouï et de proprement singulier» (12). L'expressivisme se ramifie, poursuit Taylor, en un "expressivisme de création", fondé sur le sujet individuel, et un "expressivisme de communication» (14), reposant sur le sujet collectif. Selon Larose, l'histoire récente des spéculations sur la langue au Québec est marquée par le passage de celui-ci à celui-là, de telle sorte que le syntagme «expressivisme de création» finira par apparaître comme un pléonasme. C'est du moins ce qui ressort à l'analyse - très complète - des recueils d'essais et des articles de journaux et de périodiques de l'époque.

Larose cherche à saisir le discours sur la langue en faisant entendre des harmoniques plus souterraines, plus secrètes, sous les prises de positions tonitruantes, qu'il ne veut pas complètement négliger non plus, même si c'est là que le risque de redite se révèle le plus grand. Cette position d'équilibre, pour justifiée et courageuse qu'elle soit, ne va pas sans générer son lot d'ambiguités, en particulier dans l'évaluation de l'importance de telle ou telle contribution au débat. Je trouve assez troublant le fait, par exemple, que l'auteur identifie chez Jacques Ferron les prodromes du changement de paradigme et qu'il fasse aboutir son analyse sur les prises de position de Jacques Brault alors qu'il nous dit par ailleurs que la réflexion de ces deux écrivains n'a pas trouvé l'écho mérité (pour cause d'éparpillement éditorial dans un cas, de relative confidentialité de l'œuvre dans l'autre). Tant et si bien que le récit de l'histoire en marche, me semble-t-il, entre en collision avec l'évaluation après coup et que le jugement sur la portée conceptuelle de telle pensée se superpose à l'étude de sa réception. L'histoire des marges tend ainsi à devenir l'histoire tout court ${ }^{2}$. Mais il serait injuste de ne pas signaler que Larose accorde aussi une patiente attention à d'autres acteurs dont la présence dans les débats est immédiatement remarquée, de Michel Brunet et Jean-Paul Desbiens à Gaston Miron en passant par Jean-Marc Léger, André d'Allemagne et Jean Marcel.

Le principe de l'étude de l'émergence du paradigme expressiviste étant bien établi, Larose organise sa matière, de façon tout à fait astucieuse, en quatre long chapitres qui, tout en segmentant la période, cernent certaines des inflexions majeures du débat. Le premier chapitre s'attache ainsi au passage d'une conception idéaliste du problème linguistique (du type de celle de $\mathrm{M}^{\mathrm{gr}}$ Camille Roy ou de Lionel Groulx) à une conception sociale et politique, sous l'impulsion de l'École historique de Montréal et du néo-nationalisme qu'elle promeut; le débat sur l'unilinguisme, qui constitue le nœud discursif le plus significatif de la période 1957-1963, sert opportunément de révélateur au glissement. De la perspective purement pédagogique, qui rejetait la responsabilité de la détérioration du français sur le locuteur et entendait lui inculquer la norme du bon parler, on passe, d'après Larose, à une analyse systémique du conflit et de la lutte des langues au Québec, pour finir par proposer une solution politique au problème du recul du français, dans le monde du travail tout

$$
+++
$$

2 Il faudrait s'entendre sur ce qu'est au juste la «marge » : réunit-elle les auteurs occupant une position centrale dans l'institution (Ferron, Brault, etc.), mais dont la voix est restée en retrait dans les débats de l'époque, ou les auteurs aujourd'hui considérés comme marginaux (Henri Bélanger, Giuseppe Turi, Louis Landry, etc.) qui se situaient alors au cœur des échanges? 
spécialement: l'unilinguisme institutionnel. Cette solution, bien entendu, ne s'impose pas à tous avec la même nécessité (même si elle trouve des adeptes à gauche et à droite), et le bilinguisme officiel - avec ses prolongements: le binationalisme et le biculturalisme -, notamment au fédéral, a encore de beaux jours devant lui. Parallèlement à la question de l'unilinguisme, celle de la norme mobilise les intellectuels; là se fait jour avec une particulière clarté le paradigme expressiviste qui, postulant un rapport immédiat entre la langue et l'être, lie la qualité de l'expression à l'épanouissement national de la collectivité québécoise.

Le deuxième chapitre, qui transite de la position de Ferron vers celles de Parti pris et de Liberté, se concentre sur la période 1964-1968, celle qui commence avec la généralisation de l'«équation» ferronnienne langue-littérature-politique et se termine avec la crise de Saint-Léonard, qui révèle le pouvoir déflagrateur de la "poudrière linguistique", pour reprendre l'expression de Pierre Godin. Des écrivains comme Ferron, bien sûr, mais aussi André Langevin, Laurent Girouard, André Major, Fernand Ouellette et surtout Gérald Godin apparaissent sur la scène du débat, dont l'un des enjeux principaux est le joual. Affublé, comme Janus, de deux visages, celui-ci a une face esthétique et une face politique (176). Pour les uns patois, pour les autres langue authentiquement québécoise, le joual est la ligne de faille de ces années-là, cristallisant les oppositions, forçant les uns et les autres à se (re)situer: ainsi de Major passant de pour à contre, de Michel Tremblay qui voudra échapper à l'étiquette, de Godin qui tergiversera sur la portée du phénomène, etc. Au delà de l'impasse (esthétique et politique) que représente bientôt le joual pour la plupart des intervenants, il oblige à réfléchir sur ce que pourrait devenir le français parlé et littéraire québécois. Jamais autant que durant la période 1964-1968, le rapport entre langue et littérature (et culture) n'aura donné lieu à une réflexion plus intensive.

Justement coiffé du titre "Au cœur de la poudrière linguistique», le troisième chapitre couvre la période qui va de 1968 au début des années 1970, alors que sont envisagées les premières lois linguistiques (le bill 63, la loi 22). Les positions du gouvernement Bertrand, de René Lévesque, du Front du Québec français, des syndicats, etc., vis-à-vis de la question de la langue y sont minutieusement passées en revue, délimitant l'arène d'une crise qui, pour Larose, est certes moins spectaculaire que la crise sociale, "mais tout aussi profonde», convoquant «les efforts de nombreux écrivains et intellectuels québécois autour de ce qu'on peut considérer comme la première véritable querelle du joual» (214), même si c'est en réalité la seconde. Les acteurs principaux sont ici, pour les fédéralistes partisans d'un libéralisme linguistique et d'un français québécois entièrement soumis à l'usage, Henri Bélanger et Giuseppe Turi, et, pour les indépendantistes opposés au libre jeu du français et de l'anglais comme au joual, Jean Marcel (dont la position, suivant Larose, ne semble comporter aucune faiblesse), Gilles Vigneault, Hubert Aquin, André Major, pour n'en nommer que quelques-uns. Le tableau d'époque que dessine l'auteur est particulièrement bien troussé, qui montre comment s'apostrophent et se répondent intellectuels et propagandistes, penseurs de premier plan et seconds couteaux, écrivains emportés par la déferlante (Tremblay, "caution littéraire et tête de Turc» [237], Victor-Lévy Beaulieu, l'agité de l'« ostiquement contre-culturel» [279]) et vedettes populaires prises entre l'arbre et l'écorce (Yvon Deschamps). Larose parvient à bien 
circonscrire les prises de position, faisant intervenir de manière particulièrement efficace sa grille expressivisme/utilitarisme-instrumentalisme, non sans toutefois adopter, de temps à autre, un ton un tantinet sermonneur, comme lorsqu'il distribue les bons points (à Marcel) et les mauvais (à Bélanger).

Le dernier chapitre, sur Miron et Brault, déroge quelque peu à la logique chronologique de l'ensemble. C'est manifestement lorsque Karim Larose sort du cadre et fait un pas de côté qu'il déploie toute sa compétence herméneutique. Particulièrement à l'écoute des textes, il s'écarte de la thèse pour entrer en dialogue avec des auteurs qu'il admire et qui, de toute évidence, le poussent à se surpasser. Si ce quatrième chapitre n'est pas le plus instructif de l'ouvrage, ni le mieux lié au reste du livre, c'est certainement celui où se retrouvent les plus grands bonheurs d'écriture et d'analyse. L'interprétation du "natal» — de la langue natale dans son opposition à l'idiome maternel - chez Miron et le rapprochement avec Friedrich Hölderlin sont lumineux. Sur Brault, on retient la confrontation avec l'analyse du mineur par Gilles Deleuze et Félix Guattari et la lecture très fine de «Sur le bout de la langue», essai séminal repris dans La poussière du chemin ${ }^{3}$. Les pensées de Miron et de Brault, enfin, sont elles-mêmes rapprochées et comparées, et Larose, en les faisant jouer l'une contre l'autre, ne les rêvèle que mieux.

Je n'ai pas le sentiment d'avoir rendu pleinement justice, en quelques pages, à un ouvrage foisonnant, qui n'est pas sans défauts mais qui procède d'une recherche fouillée et mobilise les ressources d'une pensée aiguisée et informée. Au final, après avoir lu La langue de papier, on voit mieux comment la littérature, qui met en jeu un "puissant expressivisme jouant sur la langue» (394), s'est révélée indispensable à la spéculation linguistique québécoise des années 1950 à 1970.

Si, au cours des années 1960, l'appel à la francophonie, comme nous le rappelle Larose, a pu servir, chez un Jean-Marc Léger notamment, à sortir le français québécois de son face-à-face inégal avec le français hexagonal et, par là, à nourrir l'utopie d'un français international, les études littéraires francophones, qui ont pris leur essor à la même époque, n'ont pas peu fait, de leur côté, pour asseoir la légitimité de la différence québécoise. Ces dernières années, les études plus transversales des littératures en français se sont multipliées et l'aire culturelle de l'Amérique francophone s'est imposée comme terrain de fertiles comparaisons: le collectif préparé récemment par Józef Kwaterko, L'humour et le rire dans les littératures francophones des Amériques ${ }^{4}$, nous en apporte encore une fois la preuve. Ce n'est pas que les études comparées y soient si présentes; mais l'analyse côte à côte des mécanismes du rire dans des littératures apparentées par la langue et par le rapport à la France fait ressortir d'intéressantes convergences, par exemple le rôle cardinal de l'auto-

$$
+++
$$

3 Jacques Brault, La poussière du chemin, Montréal, Boréal, coll. «Papiers collés», 1989. 4 Józef Kwaterko (dir.), «L'humour et le rire dans les littératures francophones des Amériques », Itinéraires et contacts de cultures, vol. 36, 2006, 198 p. 
dérision aussi bien dans l'humour des femmes au Québec (Lucie Joubert) que dans les polars africains et antillais (Ute Fendler). De même, selon Eurídice Figueiredo, les littératures de la Caraïbe francophone et de l'Amérique hispanophone et lusophone seraient caractérisées par ce «rire amer » dont parlait déjà René Ménil ${ }^{5}$ en 1945 (80). Et ainsi de suite.

Les découvertes qu'il y a à faire dans ce collectif ne concernent pas d'abord la littérature québécoise, même si le travail de Lucie Joubert est riche de perspectives prometteuses - sur l'existence d'une satire au féminin notamment - et si l'analyse de Jean-François Chassay, sur L'Immaculée Conception de Gaétan Soucy, fraye la voie à une mise en parallèle étonnante des ressorts du grotesque désespéré chez Soucy et Samuel Beckett ${ }^{6}$. Ce sont plutôt les travaux sur les littératures antillaise et acadienne qui nous réservent les plus belles surprises. On voit ainsi, avec Suzanne Crosta, comment le rire, chez Raphaël Confiant, se donne pour l'expression populaire de la sagesse (56); avec Christiane Ndiaye, on constate que l'humour discret de l'écrivaine caribéenne Gisèle Pineau doit être envisagé autrement que comme une «faiblesse» venant grever une parole féminine vouée au plus mortel sérieux. On lit aussi la belle étude, parfois poignante, sur l'humour «en mode mineur» de la romancière acadienne France Daigle: non seulement Raoul Boudreau insiste sur le fait que, chez Daigle et chez d'autres écrivains francophones du Nouveau-Brunswick, «la dérision va toujours dans le même sens et accentue la marginalité et l'étrangeté au monde des Acadiens » (131), mais il montre comment l'insécurité linguistique du minoritaire induit un retrait, un sentiment de mise à l'écart qui lui donne la distance nécessaire pour exercer son humour, à l'égard de soi bien sûr, mais également à l'endroit de l'autre: un humour dominé par la litote, l'understatement, propre à ménager entre toutes choses, pour le dire en empruntant les mots de France Daigle, «un fin passage».

$$
+++
$$

5 De René Ménil, Figueiredo cite ces phrases percutantes, qui confirment l'importance de l'autodérision chez des auteurs qui n'ont pas le statut leur permettant d'agresser l'autre par l'humour: «On voit assez que l'humour est un fusil qui part. Mais contre qui faut-il le braquer? Serait-ce, ultime geste, contre soi-même?» (81) 6 Deux articles consacrés au corpus québécois portent en tout ou en partie sur le téléfeuilleton La petite vie. On n'est plus tout à fait dans la litterrature annoncée par le titre du collectif, on en conviendra. Et si la contribution de Michèle Nevert nous perd parfois dans l'énumération encyclopédique des procédés du comique langagier déployé par Claude Meunier, celle de Benoît Melançon et Pierre Popovic, à la sociocritique aussi rapide que mordante, n'est pas loin d'adopter le ton supérieurement caustique que Denis Saint-Jacques (" "Faut voir ça" ou Liberté dans la culture de masse», Voix et Images, vol. 10, n 1, 1984, p. 141-147) avait autrefois relevé chez les intellectuels de Liberté à propos des émissions les plus populaires de la télévision québécoise. 